\title{
Research of The Influence of The Particle Geometry on The Accumulation of Repose Angle Based on DEM
}

\author{
Chen Yanhui ${ }^{1, a}$, Chu Zan ${ }^{1, b}$, Yu Xuechuang ${ }^{1, c}$ \\ ${ }^{1}$ School of Mechanical Engineering, Guangxi University of Science and Technology, Guangxi \\ Liuzhou , 545006, China; \\ agxut_jx@163.com, b605814884@qq.com, ${ }^{\mathrm{c}} 297097860 @ q q . c o m$
}

\begin{abstract}
Keywords: Grain packing; Discrete element method; Angle of repose; Matlab; Linear fitting Abstract. Particle packing is a common phenomenon in the field of industrial and agricultural, Stacking angle of repose reflects the macroscopic properties of the combined effects of granular group. In order to study the geometry of the particles impact angle of repose particle packing, in this paper, the rock particles as the research object, using discrete element software EDEM respectively simulated by a single ball, double ball, four and eight ball composed of four different geometry of rock particles in the bottomless cylinder lagged accumulation phenomenon, Combined with image processing software Matlab image of stacked linear fit contour, In non-experimental conditions measured angle of repose rock. And compared with the measured angle of repose with the actual accumulation experiments, the error is very small, and the reliability of this method is verified. The results show that: in the same condition, the different geometric shapes of the rock particles of the natural accumulation of the repose angle with the complexity of the geometric shape of the particles gradually increasing trend and reach a certain limit angle.
\end{abstract}

\section{Introduction}

Granular material is a complex system, which is formed by the interaction of a number of discrete particles, and has the characteristics of non continuous, non uniform and anisotropic and mobile. Granular materials are extremely common in nature and engineering, such as rock and soil engineering, Agricultural Sciences, pharmacology, chemical, metal, ceramics, food processing and so on. From the macro perspective, any particle materials are formed by a large number of discrete particles, in which the physical properties of each particle (such as shape, particle size and particle size distribution) and material properties (such as Poisson's ratio, elastic modulus and dynamic and static friction coefficient) are determined to a certain extent. In the last few years, researchers have paid more and more attention to the effect of the physical properties of individual particles on the micro and macro mechanical properties of the particle system [1-2]. The factors that affect the mechanical properties of granular materials such as different particle size, particle size and physical properties have been the focus of all the major projects, but at this stage, the problem has not been solved.

At present, the three main methods used in the study of the particle packing problem are Monte Carlo [3], Cellular Automation [4] and discrete element method [5]. Monte Carlo method and Cellular Automation method were used to simulate the particle packing problem from the statistical and geometrical dimensions, but does not consider real mechanical properties between the particles in the whole process of accumulation; and discrete element method truthfully reflects the particle packing process mechanical phenomenon, and it also can generate any particle size distribution, complex geometry and surface properties and a variety of materials ratio from the study sample.

The repose angle is one of the most important parameters for describing the mechanical properties of granular materials. The repose angle is the maximum angle formed by the natural slope and the horizontal plane under the condition of the natural humidity [12]. The angle of repose is important in the collapse, stratification and segregation. Angle of repose is not only dependent on the material properties of particles, such as material density, coefficient of static friction and dynamic friction coefficient; and also depends on the physical properties of the particles, such as size and geometry as 
well as a bulk forming methods. Y. C. zhou and B. H. xu et al. carried out the experimental study and numerical simulation of the glass spheres with different radius, and studied the influence of particle material properties and geometric constraints on the angle of repose by means of constructing the discrete element model considering the rolling friction coefficient [6]; Li Yan-jie et al. studied the two-dimensional packing problem of a variety of different size mixed particle system and the three-dimensional packing problem of a single size particle system [7-8]; Robinson D.A and Friedman S.P studied the effects of the particle size and the shape of the particles on the repose angle and the method of measuring the angle of repose in the process of accumulation and collapse of granular materials [9]. These studies are mostly from the type of granular material or replaced the primary particles with spherical particles to be analyzed, and did not consider the complex mechanical properties of the particles packing within the body structure, but for different geometric shapes, sizes and grading and other factors non-uniform discrete particles accumulate practical problems also consistent with the lack of systematic research and analysis.

This paper is based on the method of the irregular geometric shape of the particles [10-11], and the material of the rock is the research object. The numerical simulation of rock particles with different geometric shapes is realized by using the discrete element software EDEM. The numerical simulation is carried out with the image processing software Matlab, and the effect of different geometrical shapes on the natural accumulation of the natural accumulation of the natural deposit is studied. The results show that: in the same conditions, the more irregular geometry of rock particles, the greater its natural angle of repose accumulation and reaches a certain limit angle.

\section{Discrete Element Method Introduction}

The discrete element method (discrete element Method) "the first is by Minnesota University Cundall P.A professor put forward at the beginning of the seventies of the 20th century. Originally, it is mainly used for the study of rocks and other non continuum mechanics behavior.

\section{Basic principle of DEM}

The basic principle of the discrete element method is to consider the discrete system as a collection of certain mass and shape of the discrete particle element, which gives the contact between the particles and the contact boundary. The discrete element method is used to calculate the contact force between the particles and the speed and position of each particle, and then the whole particle system. The method is used to solve the problem. In the discrete element method, the interaction between particles is considered as a dynamic equilibrium, which can be considered as the equilibrium state when the forces acting on the system are stable.

\section{Basic algorithm of DEM}

The discrete element method calculation circulation process: first, through the force and displacement between particles or the relationship between the displacement increment, determine the size of the force F; Then using formula (Newton's second law), to determine the particle acceleration; To integral of acceleration, again can get the displacement $\mathrm{S}$ particles. According to the process cycle and traverse the entire particle set, which can calculate each force of particles in different time, the information such as acceleration, velocity and displacement. The calculation process is shown in Fig. 1.

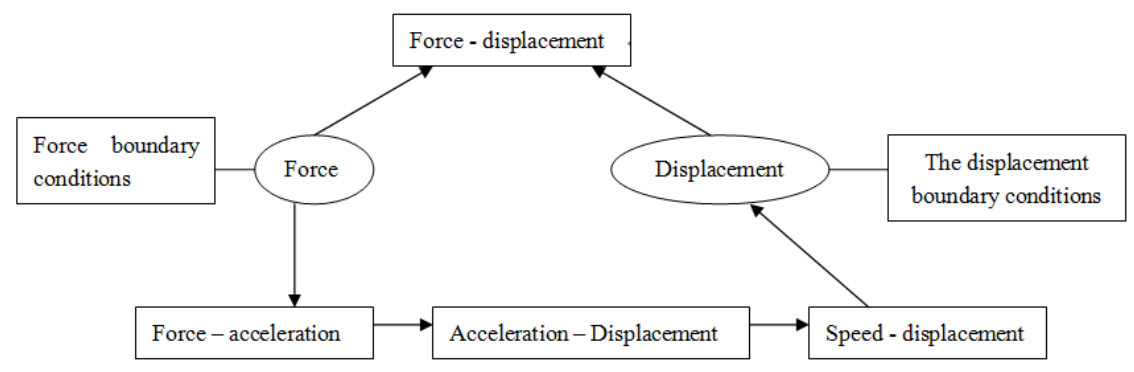

Fig. 1 Force-displacement calculation cycle diagram 


\section{Particles Numerical Simulation of Natural Accumulation}

\section{Setting parameters, physical and material properties}

Rock particles as the research object of the text, since the particle size is not large, bottomless cylinder method is used when using the discrete element software EDEM accumulation of particulate materials can be simulated [12]. In the EDEM, the material properties and the contact properties of the rock particles and the non bottom cylinder need to be considered in the model. the material parameters are density, poisson's ratio and shear modulus, and the contact parameters that need to be set are the coefficient of restitution, static friction coefficient and dynamic friction coefficient. Refer to the relevant literature and refer to the results of previous studies, the simulation parameters of rock in the EDEM are set to Table 1 and Table 2 [13-16].

Table 1 Material properties table

\begin{tabular}{cccc}
\hline Material & Poisson's ratio & Shear modulus $(\mathrm{Pa})$ & Density $\left(\mathrm{kg} / \mathrm{m}^{3}\right)$ \\
\hline Rock & 0.2 & $5 \mathrm{e}+07$ & 2600 \\
Steel & 0.3 & $7 \mathrm{e}+10$ & 7800 \\
\hline \multicolumn{5}{c}{ Table } & 2 Contacts attribute table \\
\hline Material & Coefficient of Restitution & Coefficient of Static Friction & Coefficient of Rolling Friction \\
\hline Rock - Rock & 0.5 & 0.9 & 0.15 \\
Rock - Steel & 0.5 & 0.4 & 0.05 \\
\hline
\end{tabular}

\section{Modeling of rock particles}

In order to realize the three-dimensional discrete element modeling of irregular geometry of rock, in this paper, the rock as the research object, and using the V5 Scanworks laser scanning probe to scan the surface of rock, and then using Studio Geomagic software to obtain the 3D geometry boundary model of triangular surface mesh with high accuracy and good quality. In the EDEM, the 3D discrete element model of the rock particles with irregular geometry is realized by using the method of superposition method in the inner region of the geometrical boundary of rock. The discrete element modeling process of rock particles is shown in Fig. 2.

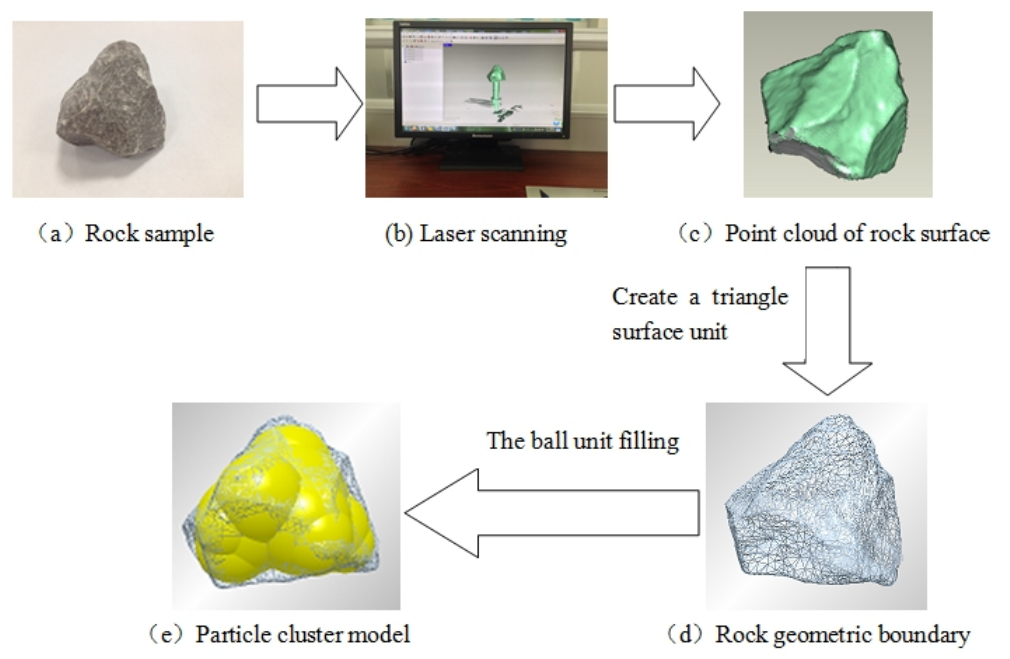

Fig. 2 Rock particles discrete element modeling process

When the particles are modeled in EDEM, the particle geometric shape and size can be changed by controlling the number of spherical particles, the size of the particles and the different combinations. Using EDEM software is quick and convenient to generate different types of particle clusters, the more the number of particle clusters basic ball is, the more features of the geometry of rock can be described, its numerical simulation results and the actual situation is more closer, but the calculation amount corresponding increase. In order to reduce the computation time, we need to balance the 
computational efficiency and rationality of the calculation results by simplifying ball composition unit in the actual calculation. Mc Dowell et al. believes that when the ball combination unit basic ball number greater than 8 , it can be a true reflection of particles between the occlusion effect [17]. So here we have a single ball, double ball, four-ball and eight ball, respectively, for the same particle-filled rock geometric boundaries to obtain four different geometric shape of the particle clusters, shown in Figure 3. In the EDEM, the four kinds of particles are used to simulate the particle model, and then the bulk properties are compared.

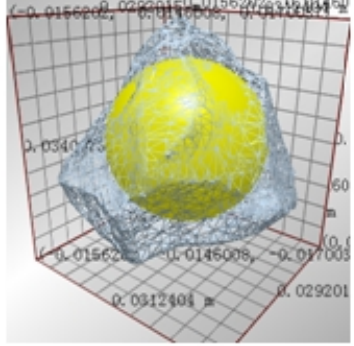

One ball

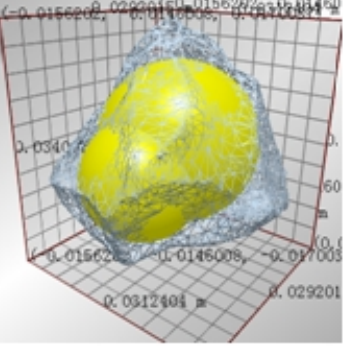

Two balls

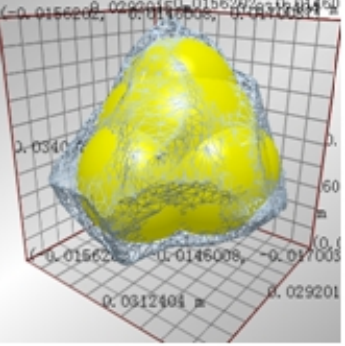

Four balls

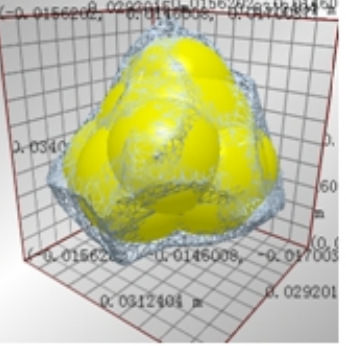

Eight balls

Fig. 3 The four models of particles in simulation

\section{Select contact model}

The discrete element contact model describes the behavior of the elements, and the appropriate discrete element contact model has a very important influence on the simulation results. The EDEM mainly includes the following integrated contact model: A Hertz-Mindlin contact model without sliding, Ç Hertz-Mindlin bond model, É Hertz-Mindlin heat conduction model, Ñ Temperature Update (temperature update), (5) Linear Cohesion (linear bond) contact model, (6)Linear Cohesion (linear spring) model, (7)Moving Plane (movement plane) contact model, 8)Tribocharging (triboelectrification) exposure models, different contact model their scope varies. Because of the relatively low water content of rock particles, the particles can be considered as an ideal particle, so the contact model of Hertz-Mindlin is suitable for this paper [13].

\section{Geometry modeling and rock forming}

(1) Geometry Modeling. The size of the bottomless cylinder are determined by the size of the particle size. Practice shows that the bottomless cylinder diameter and height of the ratio of 1:3, bottomless cylinder diameter should be greater than 4 to 5 times the maximum particle size of particles. Because the average size of the rock particles is $25 \mathrm{~mm}$, the diameter is $130 \mathrm{~mm}$, the height is $390 \mathrm{~mm}$. Using stainless steel material in accordance with the above parameters to establish a bottom cylinder model. The main purpose of this paper is to simulate the accumulation process of rock particles in the stainless steel plate. The contact parameters of rock and stainless steel are known. Therefore, the stainless steel is a material which is in contact with the bottom of the base material. The bottom plane is parallel to the stainless steel plate.

(2) Rock forming. Bottomless cylinder generated after, the dynamic particle generation method is used to generate the rock particles (eight ball particle cluster model as an example) in "Factories". When the rock particles are filled with a cylinder, a cylinder is given at a slower constant speed and the vertical direction of the plate is vertically upward. See Figure 4. 


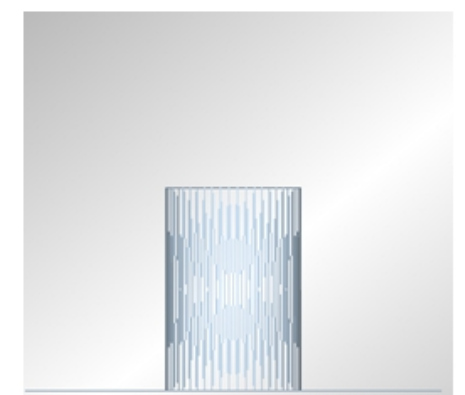

a. Bottomless cylinder body modeling

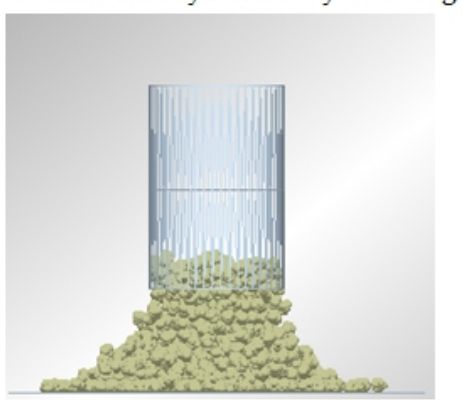

c. Rock particle accumulation process

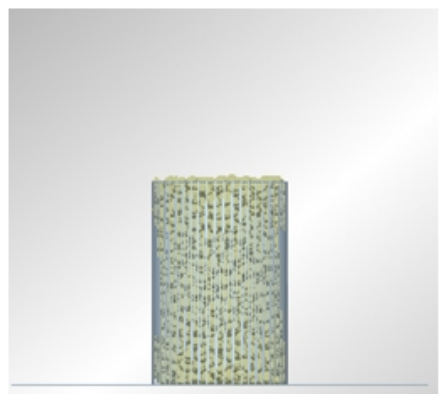

b. Filling with rock particle

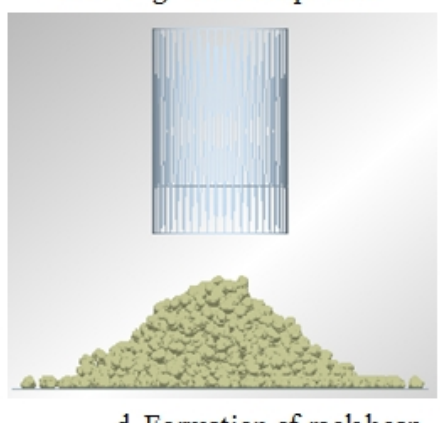

d. Formation of rock heap

Fig. 4 Accumulation simulation of rock particle

(3) Determine the simulated conditions. When bottomless cylinder are adopted to simulate the rock particles accumulation process, not only the bottomless cylinder specification and rock particle shape and material properties will affect the particle accumulation form, but also different lifting speed of the bottomless cylinder will have a greater impact on the accumulation of particles form. To avoid the large stock pile forming in the process of diffusion, which may effect the precision of particle repose of angle, in this paper, the lifting speed is $0.05 \mathrm{~m} / \mathrm{s}$ proposed by Li Qin-liang [18]. At this rate, the formation of the material pile is complete and the edge diffusion is not obvious.

\section{Repose of angle measurement}

(1) The simulation of the repose angle. After the formation of the rock and stone, the image processing software Matlab was used to measure the angle of repose. Concrete steps: (a) the data is divided into two parts, the Matlab software is used to read the data of the left and right sides of the data, and the gray image is displayed; (b) to the left and right sides of the rock and stone image of binarization operation, and left and right sides of the image of the boundary contour search. (c) the slope boundary of the material pile is selected, and the linear fitting is obtained. The slope of the equation can be obtained by the linear fitting equation, $\mathrm{K}$. Then the repose of angle can be obtained by the following formular [19]:

$$
\theta=\frac{\arctan |k| \times 180^{\circ}}{\pi}
$$

Where: $\theta$ is the angle of repose of rock particles measured values, the unit is ${ }^{\circ} ; \mathrm{k}$ is the slope of the linear regression equation.

(2) Experimental measurement of the repose angle. In the experiment, we used the conventional method to measure the angle of repose of the rock, in order to get the average value of rock pile height $\mathrm{h}$ and base diameter $\mathrm{d}$ with the way of multi-angle, multi-frequency, and the rest angle of the rock was calculated by the following formula:

$$
\theta^{\prime}=\arctan \left(\frac{2 h}{d}\right)
$$

Where: $\theta$ 'experimental measurements of rock particles angle of repose, the unit is ${ }^{\circ} ; \mathrm{h}$ for the rock pile the stack height, the unit is $\mathrm{cm}$; $d$ is the diameter of the bottom surface of the rock pile, the unit is $\mathrm{cm}$. 


\section{Simulation results and analysis}

Figure 5 is the use of image processing software Matlab to stockpile about side angle of repose of the measurement process, it can be seen from Figure 6, using the least squares method on rock stockpile unilateral linear fit contour, resulting equation is: $\mathrm{y}=0.79675 \mathrm{x}+471.2857$, the slope $\mathrm{k}=0.79675$, from equation (1) calculated angle of repose is $38.55^{\circ}$. Equation $\mathrm{x}$ represents the horizontal pixel image, $y$ represents the vertical pixels of the image are no real dimension to the pixels of the image for the establishment of a Cartesian coordinate system axes, as shown in Fig. 5c, the linear regression equation can be Characterization of linear trend image contours well, but the slope is obtained by the formula $\mathrm{k}$, just to accurately reflect the practical significance of the accumulation of angular size.

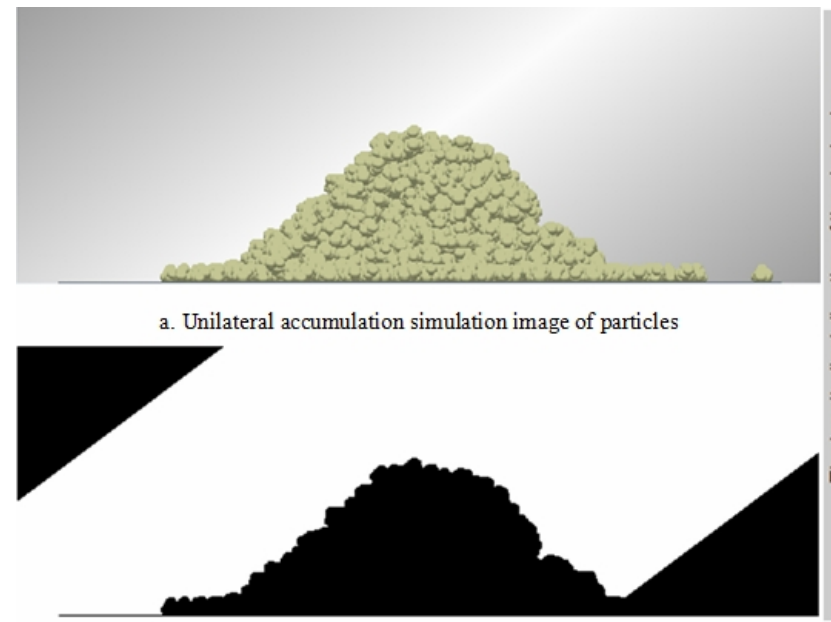

b. Unilateral accumulation binary image of particles

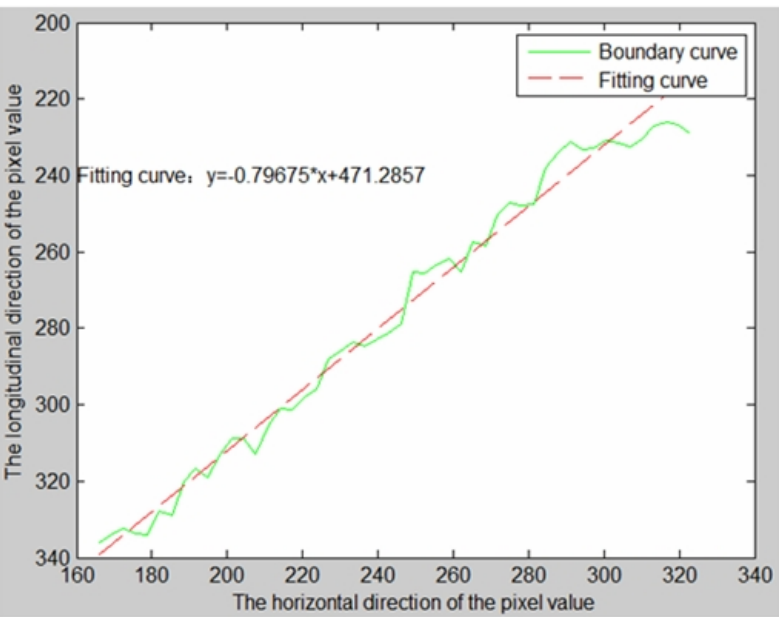

c. Unilateral contour line and linear fitting diagram of particles

Fig. 5 Numerical measurement of repose angle of rock particle

By using this method, the calculated values of the repose angle of the 6 repeated simulation of rock particles are: $38.55,38.19,37.86,38.63,38.02$ and 37.94 respectively. The mean value is 38.22 , and the standard deviation is 0.32 . Thus, for the rock particles of the eight spheres, the discrete element method is used to simulate the accumulation, and the accumulation angle is 38.22 degrees 0.32 .

\section{Experiments}

In order to further verify the validity and accuracy of the method of measuring the angle of repose, real rock particles and stainless steel bottomless cylinder are used in the experiment. The rock particles used in the experiment need to be carefully screened and selected, and the particle size and particle shape are close to 4 kinds of particle model in simulation. In order to make the number of rock particles loaded in the bottom of the cylinder with the same size, the number of particles in the bottomless cylinder which was calculated by analyst software of the EDEM is 1200. The total mass is $28.50 \mathrm{~kg}$, in the experiment of quality as the standard. Experiments will be subject to the quality, the use of electronic balance said learn artificial screening selected rock materials $28.50 \mathrm{~kg}$. In order to ensure that the speed of the cylinder is $0.05 \mathrm{~m} / \mathrm{s}$ in accordance with the simulation condition, through the stepping motor to drive the pulley block, the slow speed of the bottom cylinder is slowly upward, so as to reduce the experimental error caused by the jitter or eccentricity in the process. Fig. 6 experiment and simulation comparison chart, from stock pile form, two were closer, but both accumulation figure on the smoothness of the slope is slightly different, mainly because the particles in the simulation process is composed of ball, and the appearance is smooth, and experimental rock particles although the screening, but shape is very irregular, with many edges and corners. 


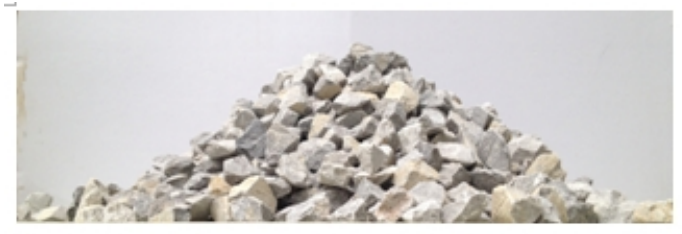

a. Experimental verification

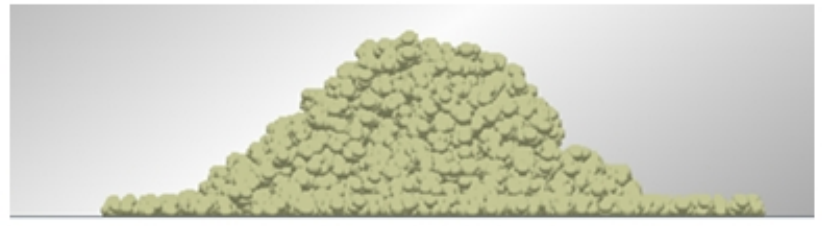

b. Experimental simulation

Fig. 6 Experimental verification and simulation of rock particles' accumulation

The experimental scheme is adopted. The experiment was repeated six times respectively, the accumulation angles which is given by the formula (2) were $38.23^{\circ}, 37.54^{\circ}, 38.76^{\circ}, 38.07^{\circ}, 37.15^{\circ}$ and $38.52^{\circ}$. The mean value is 38.05 , and the standard deviation is 0.55 . Therefore, the angle of repose of the raw material is $38.05^{\circ} \pm 0.55^{\circ}$. Repose of angle error and analog measured $0.45 \%$. Therefore, it can be explained by using the discrete element method combined with the image processing software Matlab to measure the angular accuracy of the particle material.

\section{All simulation results and analysis}

By comparing the simulation results in Table 3, we can see that the angle of repose of the three kinds of particles with double ball, four ball and eight ball is obviously larger than that of the single pellet. The main reason is that the more complex the particle geometry, the more contact number of the particles in the material pile, and the particles in the force chain are more likely to produce a self locking phenomenon, which limits the movement of the particles. In nature and engineering, the bulk material is in the form of irregular polyhedron. In this simulation, the particles contact with the eight spheres, which are similar to the irregular polyhedron. The support effect is the strongest, and the movement of the particles is hindered by the geometrical shape of the particles. Single spherical particles, double ball particles and four spherical particles are more smooth and less contact with the number of particles, and can not truly reflect the contact between the edges and corners of the rock grains, and the contact of the angle and the angle. Although the number of basic spheres in the particle cluster is more and more, the more can describe the geometrical shape of the rock, and the numerical simulation results are close to the actual situation. In order to reduce the computation time, we need to simplify the calculation of the particle cluster to balance the calculation efficiency and the rationality of the results. MC Dowell believes that when the ball combination unit basic ball number greater than 8 , can be a true reflection of particles between the occlusion effect.

Table 3 Simulation results

\begin{tabular}{lcccc}
\hline Simulation data & Single ball & Two balls & Four balls & Eight balls \\
\hline Angle of repose ${ }^{\circ}$ & $32.68^{\circ}$ & $34.62^{\circ}$ & $36.39^{\circ}$ & $38.55^{\circ}$ \\
Contact number & 9068 & 10937 & 11836 & 12501 \\
\hline
\end{tabular}

It can be seen that the contact impact between particles is closely related to the shape of the particles. When we need to simulate the complex shapes of particles in the simulation, the simulation results may be inaccurate.

\section{Conclusions}

In this paper, the discrete element method is used to simulate and analyze the particles in the bulk state. Under the premise of the physical parameters of the material and the material parameters of the material are determined, the material accumulation image was obtained by using the discrete element software EDEM and the image processing software Matlab was combined with the image processing software. In this paper, the results of the numerical method for the measurement of the angle of repose are in agreement with the experimental results. On the basis of the above, the influence of the geometrical shape of the particles on the repose angle of the particles is studied in this paper. The results show that: as the particles form from the smooth to irregular changes in the irregular edges and corners, and then lead to the bite force of the particles to strengthen, and the movement of the particles 
is improved, so the different forms of the different forms of the angle of repose are gradually increasing and reaching a certain limit.

\section{Acknowledgements}

This work was financially supported by Guangxi science and technology development program (No. 1598021-2), Education department of Guangxi key project (No.ZD2014074), the Guangxi Natural Science Foundation (No. 2015GXNSFAA139271) and Doctoral foundation of Guangxi University of science and technology (No. 13Z13).

\section{References}

[1] KUHN M R. Heterogeneity and patterning in the quasi-static behavior of granular materials [J].Granular Matter, 2003, 4(4):155-166.

[2] Antony, S. J, Kuhn, M. R. Influence of particle shape on granular contact signatures and shear strength: new insights from simulations [J]. International Journal of Solids and Structures, 2004, 41: 5863-5870.

[3] Elperin, T, Vikhansky, A. Stress distribution in sandpiles-A variational approach [J]. Physica A, 1998,260:201-217.

[4] Mehta. A, Barker, G. C. Disorder, memory and avalanches in sandpiles [J]. Europhysics Letters, 1994,27: 501-506.

[5] Cundall, P. A, Strack, O. D. L. A discrete numerical model for granular assemblies [J]. Geotechnical Engineering, 1979,29:47-65.

[6] Zhou, Y. C, Xu, B, H, Yu, A. B, etal. An experiment and numerical study of the angle of repose of coarse spheres [J]. Powder Technology, 2002, 125:45-54.

[7] Li Yan-jie, Xu Yong .Particle packing problem with a discrete element simulation [J] Agricultural Mechanization Research, 2005,2: 57-59.

[8] Yanjie Li, Yong $\mathrm{Xu}$, Colin Thornton. A comparison of discrete element simulations and experiments for 'sandpiles' composed of spherical particles [J].Powder Technology, 2005, 160:219-228.

[9] Robinson D. A, Friedman, S.P. Observations of the effects of particle shape and particle size distribution on avalanching of granular media. Physica A.2002,311(14):97-110.

[10] Cheng Si-yuan, Xie Shao-wang. Geomagic Studio reverse engineering technology and its application [M] Beijing: Tsinghua University Press, 2010.

[11] Hu Ying-feng. Reconstruction after reverse engineering [J] Processing Surface-point line based mining machinery, 2008,29 (6): 201-202.

[12] Wu Ai-xiang, Sun Ye-zhi, Liu Xiang-ping. Dispersion dynamics theory and its application [M] Beijing: Metallurgical Press, 2002.

[13] Wang Guo-qiang, Hao Wan-jun, Wang Ji-xin. Discrete element method and its practice in the EDEM's [M] Xi'an: Northwestern University Press, 2010: 4-5.

[14] Yang Yang, Tang Shou-gao. Selection Method particle discrete element method, damping, stiffness coefficient and time step [J]. Computer Aided Engineering, 2007,16 (3): 65-68.

[15] Yan Ying, Ji Shun-ying. Numerical simulation of a combination of particle aggregates direct shear cell [J]. Journal of Applied Mechanics, 2009,26 (1): 1-7. 
[16] Zhang Guang-cheng, Xiang xin. Rockfall restitution coefficient of field tests and numerical calculation [J]. Rock Mechanics and Engineering, 2011, 30 (6): 1266-1273.

[17] Ferellec J.F, McDowell G. R. A simple method to create complex particle shapes for DEM [J]. Geomechanics and Geoengineering, 2008, 3(3):211-216.

[18] Li Qin-liang DEM stacked nature of the particles and bulk materials reproduced Process Simulation Study [D] Shenyang: Northeastern University, 2010.

[19] Lleleji KE, Zhou B. The angle of repose of bulk co rn stover particles [J]. Powder Technology, 2008, 187(2): 110-118. 